\title{
Buchbesprechung/Book Review
}

\author{
Methods in Molecular Biology 520 \\ Tumor Biomarker Discovery - Methods and Protocols \\ Michael A. Tainsky (Editor) \\ Humana Press/Springer Verlag GmbH 2009 \\ ISBN: 978-1-60327-810-2 \\ DOI: $10.1007 / 978-1-60327-811-9$ \\ Preis: $€ 80,20$
}

Allein die eindrucksvolle Zahl von 520 bisher erschienenen Bänden der ,Methods in Molecular Biology“ " belegt den Wert und die Beliebtheit dieser Serie von Humana Press (seit 2006 eine Tochter des Springer Verlags). Rechnet man die übrigen Humana-Buchserien wie Molecular Medicine, Clinical Pathology oder Clinical Oncology dazu, dann kommt man auf über 1.500 Bände, erschienen über einen Zeitraum von 32 Jahren. Keine andere medizinische Buchserie kann eine vergleichbare Bilanz und Beständigkeit vorweisen.

Wer die Serie noch nicht kennt, sollte wissen: ,Tumor Biomarker Discovery " ist kein Lehrbuch im landläufigen Sinne, sondern eine Sammlung von erprobten und kommentierten Kochrezepten für Analytiker. Auf das Milligramm genau ist hier nachzulesen, was man vorbereiten muss, um Biomarker für die Krebsdiagnostik zu entdecken, seien es zirkulierende DNA-Moleküle, Autoantikörper oder Hormonprofile. Potenzielle Leser sind Wissenschaftler, die sich für die Identifizierung und Entwicklung neuer Tumorbiomarker interessieren sowie Vertreter diagnostischer Disziplinen, die sich mit den neuen Techniken vor ihrem Eintritt in die klinische Praxis vertraut machen wollen.

Der Herausgeber Michael Tainsky (Detroit) beginnt mit einer fast trivialen Definition, die es in sich hat: ,,Biomarker sind molekulare Indikatoren für einen biologischen Status. Sie dienen der biochemischen Evaluation von Kranheitsstatus und therapeutischen Interventionen. " Gilt das nicht für alle längst bekannten Tumormarker, wenn nicht sogar für alle Analyte der Labordiagnostik?

Völlig korrekt: Klinische Chemie und Laboratoriumsmedizin befassen sich seit jeher mit Biomarkern, und doch haben neue Technologien deren Einsatz in letzter Zeit grundlegend verändert. Während man früher einem einzigen Analyten ein ganzes Forscherleben widmen konnte, ermöglichen es die in diesem Buch vorgestellten Verfahren, große Sub- stanzgruppen mit Hunderten oder Tausenden von Mitgliedern gleichzeitig $\mathrm{zu}$ erfassen. $\mathrm{Ob}$ es sich nun um 2D-Elektrophorese oder Bead-basierte Multiplex-Immunoassays, DNA-, Protein oder Gewebe-Array, Massen- oder Kernresonanz-Spektroskopie handelt, stets erhält der Untersucher als Ergebnis eine Datenmenge, gegen die noch so umfangreiche Profile herkömmlicher Tumormarker recht bescheiden wirken. Ein eigenes Kapitel widmet sich deshalb auch der Entwicklung und Bewertung multivariater Klassifikatoren für die Krebsdiagnostik - Verfahren also, die große Zahlenmengen zu einer einzigen, aussagekräftigen Maßzahl zusammenfassen.

Insgesamt 62 Autoren, durchweg aus US-amerikanischen und zum Großteil universitären Einrichtungen, teilen in diesem Buch ihre Fachkompetenz mit den Lesern. Die meisten der 22 Kapitel beschreiben eine ganz bestimmte Biomarkerklasse (z.B. Serumproteine) und eine ganz bestimmte Technologie (z.B. Massenspektrometrie), in der Regel auch nur das System eines ganz bestimmten Herstellers. Umfassende Literaturlisten ermöglichen gezielte weitere Recherchen.

Jedes Kapitel steht für sich allein und kann ohne Kenntnis des übrigen Inhalts gelesen werden. Die Anordnung ist recht willkürlich; hier wäre mit Sicherheit eine ausführliche Einleitung des Herausgebers, wie sie in anderen Bänden der Serie geboten wird, nützlich gewesen. So muss man sich mit zwei Seiten Vorwort begnügen, das aber auch nicht viel mehr bietet als das Inhaltsverzeichnis, und auch das Stichwortverzeichnis hilft nur bedingt bei der Orientierung.

Dafür vermittelt aber die Lektüre der Einführungen zu den einzelnen Kapiteln einen schnellen und trotzdem tiefen Einblick in die Welt der onkologischen Biomarker. Für eine erste Übersicht muss man nicht einmal das ganze Buch kaufen, denn die Zusammenfassungen stehen im Internet unter www.springerprotocols.com. Es genügt, die obige DOINummer bei Google einzugeben, um kostenlosen Zugang zu den Abstracts zu erhalten und sich bei Interesse einzelne Kapitel gegen Gebühr herunter zu laden.

Georg Hoffmann (Grafrath) 